\title{
CONASIST C2015, NUEVA VARIEDAD DE TRIGO MACARRONERO (Triticum durum Desf.) PARA SIEMBRAS DE RIEGO EN MÉXICO
}

\author{
CONASIST C2015, A NEW VARIETY OF DURUM WHEAT \\ (Triticum durum Desf.) FOR PLANTING UNDER IRRIGATION IN MEXICO
}

\begin{abstract}
René Hortelano-Santa Rosa ${ }^{1}$, Héctor E. Villaseñor-Mir ${ }^{1 *}$, Julio Huerta-Espino ${ }^{1}$, Eliel Martínez-Cruz ${ }^{1}$, Ernesto Solís-Moya ${ }^{2}$, Jorge I. Alvarado-Padilla ${ }^{3}$, Alberto Borbón-Gracia ${ }^{4}$, Eduardo Espitia-Rangel ${ }^{1}$, Gabriela Chávez-Villalba ${ }^{4}$, Héctor Cortinas-Escobar ${ }^{5}$, Juan Martínez-Medina ${ }^{6}$, Leodegario Osorio-Alcalá7, María F. Rodríguez-García' y Javier Ireta-Moreno ${ }^{8}$
\end{abstract}

\begin{abstract}
'Instituto Nacional de Investigaciones Forestales, Agrícolas y Pecuarias (INIFAP), Campo Experimental Valle de México, Coatlinchán, Texcoco, Estado de México, México. ${ }^{2}$ INIFAP, Campo Experimental Bajío, Celaya, Guanajuato, México. ${ }^{3}$ INIFAP, Campo Experimental Valle de Mexicali, Mexicali, Baja California, México. ${ }^{4}$ NIFAP, Campo Experimental Norman E. Borlaug, Ciudad Obregón, Sonora, México. ${ }^{5}$ INIFAP, Campo Experimental Río Bravo, Rio Bravo, Tamaulipas, México. ${ }^{6}$ NIFAP, Campo Experimental General Terán, General Terán, Nuevo León, México. ${ }^{7}$ INIFAP, Campo Experimental Valles Centrales de Oaxaca, Santo Domingo Barrio Bajo, Etla, Oaxaca, México. ${ }^{8}$ INIFAP, Campo Experimental Centro Altos de Jalisco, Tepatitlán de Morelos, Jalisco, México.
\end{abstract}

*Autor de correspondencia (villasenor.hector@inifap.gob.mx)

México ha sido deficitario en el abasto de trigo panificable (Triticum aestivum L.), situación que se ha agravado en épocas recientes, ya que la superficie sembrada con este cereal ha disminuido en $32 \%$ de 1985 a la fecha (INEGI, 2017); por el contrario, la producción de trigo cristalino o macarronero (Triticum durum Desf.) es suficiente para cubrir la demanda nacional y exportar los excedentes.

Uno de los problemas fitopatológicos principales del trigo cristalino es la presencia de nuevas razas de roya de la hoja (Puccinia triticina E.) y de roya amarilla (Puccinia striiformis f. sp. tritici), que han causado pérdidas considerables en el noroeste y norte del país, tal es el caso de la raza BBG/ BNG que en el año 2001 venció la resistencia de Altar C84, lo que originó pérdidas por $\$ 802$ millones de pesos $M X$; las razas BBG/BPC y CBG/BPC sobre las variedades Júpare C2001, Samayoa C2004 y Banámichi C2004, que en el año 2008 causaron pérdidas por $\$ 508$ millones de pesos MX (Huerta-Espino et al., 2009) y en el año 2016 la raza BBG/BPC que venció la resistencia de Cirno C2008 (PérezLópez et al., 2017).

El impacto negativo de la evolución de las razas sobre el rendimiento del trigo se ha controlado genéticamente con la liberación continua de nuevas variedades; sin embargo, los hongos siguen presentando nuevas variantes que rompen la resistencia de las variedades. Conasist C2015 es una nueva variedad que el Instituto Nacional de Investigaciones Forestales, Agrícolas y Pecuarias (INIFAP) pone a disposición de los agricultores de trigo de riego en México, con resistencia a las razas de roya de la hoja y roya amarilla que prevalecen en el país.
La línea experimental que dio origen a Conasist C2015 se generó en el Programa de Mejoramiento Genético de Trigos Macarroneros del CIMMYT y posteriormente se evaluó en los ensayos nacionales de trigo de riego del INIFAP. La línea provino de la recombinación de una cruza trilineal entre los parentales A (TRN//21563/AA/3/BD2080/4/ BD2339), B (RASCON37/TARRO2//RASCON37) y C (AUK/ GUIL//GREEN); posteriormente, se efectuó selección familial y masiva, hasta obtener la generación $\mathrm{F}_{7}$ en la que se seleccionó la línea experimental cuya genealogía es la siguiente:

\section{TRN//21563/AA/3/BD2080/4/BD2339/5/RASCON37/ TARRO2//RASCON37/6/AUK/GUIL//GREEN}

Las selecciones se efectuaron alternadamente en el Campo Experimental Norman E. Borlaug en el Valle del Yaqui, Sonora (Y) (270 22' 12" N, $109^{\circ} 55^{\prime}$ 51" O, 40 msnm), El Batán, Estado de México (B) (19³2' 41" N, $98^{\circ}$ 51' 18" O, 2249 msnm) y San Antonio Atizapán, Estado de México (M) (190 13' 38" N, 99 33' O, 2581 msnm). La generación $\mathrm{F}_{7}$ se cosechó masivamente como línea uniforme en el Valle del Yaqui durante el ciclo otoñoinvierno/2003-04 y se reconoció con el siguiente pedigrí: CDSS00B00364T-0TOPY-0B-2Y-0M-0Y-1B-0Y.

La línea experimental se empezó a evaluar a partir del ciclo otoño-invierno/2007-08 por el INIFAP en sus ensayos nacionales en 98 condiciones diferentes de producción de 10 entidades federativas, así como en viveros de evaluación para enfermedades en los Valles Altos de México en los ciclos primavera-verano/2011 a 2015. En las evaluaciones, 
la variedad Conasist C2015 superó el rendimiento de grano a las variedades testigo, mostró mayor resistencia a enfermedades y su calidad satisfizo los requerimientos de la industria semolera nacional.

Conasist C2015 cuenta con el registro (TRI-175-231117) en el Catálogo Nacional de Variedades Vegetales (CNVV) del Servicio Nacional de Inspección y Certificación de Semillas (SNICS) y con el Título de Obtentor Número 1896.

Esta variedad es de hábito de primavera, de ciclo intermedio (122 días promedio a madurez fisiológica), porte medio ( $87 \mathrm{~cm}$ de altura de planta) (Figura 1), tolerante al acame, resistente a roya de la hoja (con valores de 0 a 10R) y a roya amarilla (de 0 a 10MR), con alto potencial de rendimiento en riego normal (cuatro riegos de auxilio) y riego limitado (tres riegos de auxilio, con estrés hídrico en la etapa de llenado de grano). Presenta muy fuerte coloración de antocianinas en el coleóptilo y muy baja o ausente frecuencia de plantas con hoja bandera curvada; tiene espiga de color blanco, barbas de color blanco, con densidad media y longitud mediana. La gluma en el tercio medio de la espiga es de forma oblonga media, con hombro de forma puntiaguda y de anchura estrecha; su pico es de longitud corta, de curvatura débil y sin de vellosidad en la superficie externa. El grano es color ámbar (Figura 2), de forma ligeramente alargada, con longitud corta de los pelos del pincel y de coloración ligera al aplicarle fenol.

El rendimiento de grano de Conasist C2015 es mayor

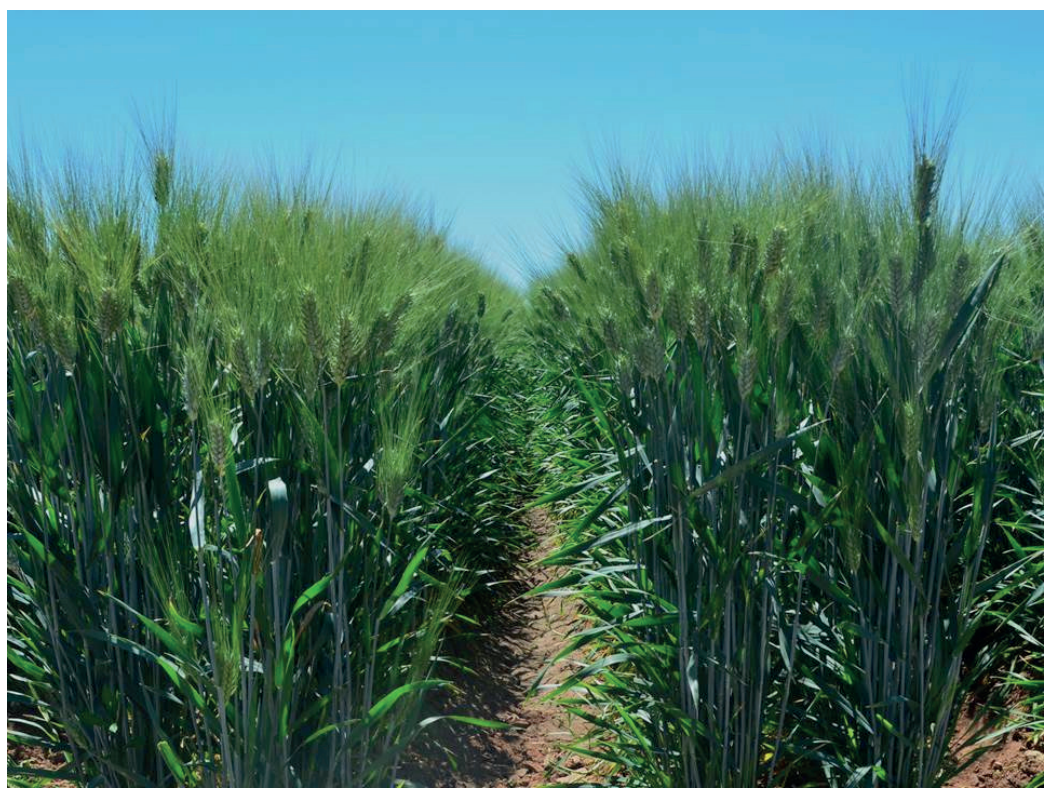

Figura 1. Porte de planta de la variedad de trigo macarronero Conasist C2015

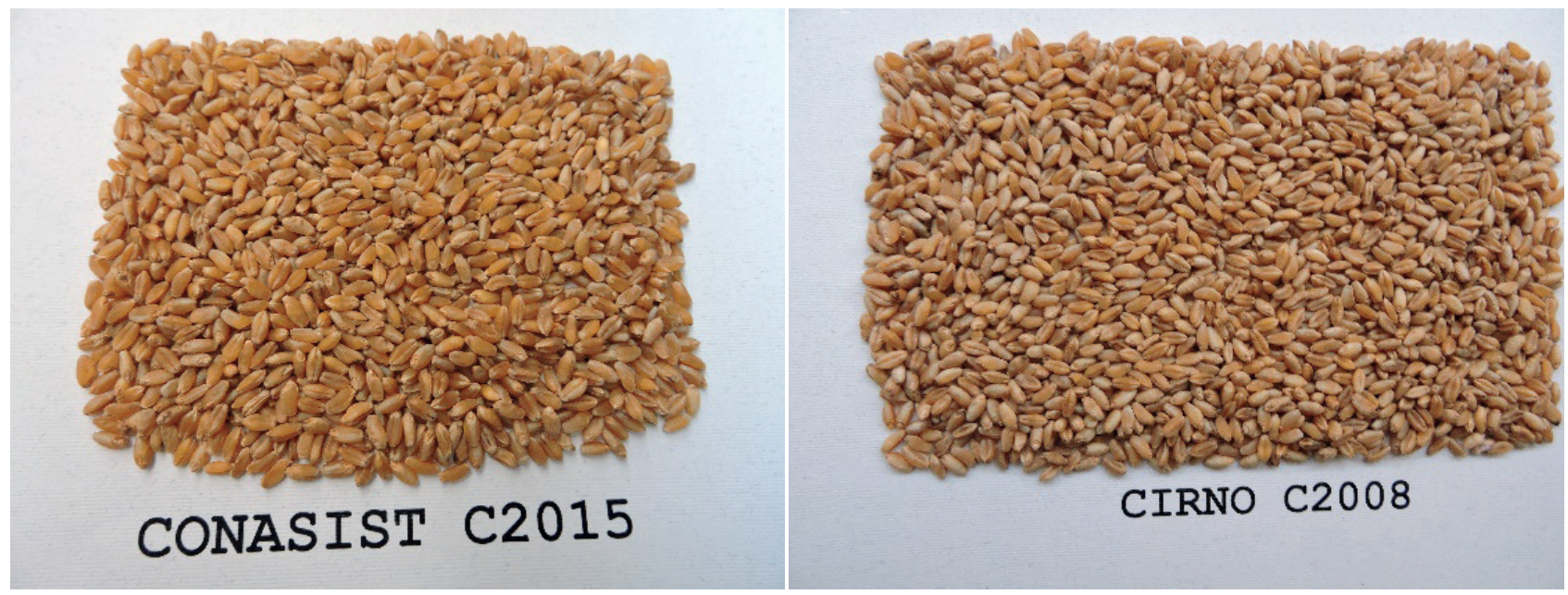

Figura 2. Granos de las variedades Conasist C2015 y Cirno C2008. Es notoria la mayor pigmentación de Conasist C2015. 
(Cuadro 1) que el de las variedades testigo con las que se comparó (Anatoly C2011, Cirno C2008, Gema C2004, Río Colorado C2000 y Movas C2011), superándolas de 10.0 (Anatoly C2011) a 14.5 \% (Movas C2011), en riego normal y de 2.3 a $13.7 \%$ en riego limitado. Además, su rendimiento es más estable y posee mayor resistencia a las razas de royas de la hoja y roya amarilla que prevalecen en las áreas productoras de trigo en México. El grano de esta nueva variedad presenta mayor peso hectolítrico (80 kg $\mathrm{hL}^{-1}$ ) que los testigos, lo que incrementa el rendimiento de sémola; su grano vítreo está asociado con un mayor color amarillo de su sémola, lo que favorece la calidad de pastas y macarrones.

Conasist C2015 se recomienda para áreas de riego del noroeste, norte y noreste de México, en fechas de siembras tempranas a tardías; en El Bajío responde bien en fechas de siembras tempranas a intermedias. El programa de trigo del INIFAP-CEVAMEX resguarda y pone a disposición de partes interesadas las categorías de semilla original y básica de esta variedad.

\section{AGRADECIMIENTOS}

Al Programa de Trigos Macarroneros del CIMMYT por la generación de la línea y al FONSEC SAGARPA-CONACYT (Proyecto 146788) por el financiamiento de los ensayos nacionales.

\section{BIBLIOGRAFÍA}

INEGI, Instituto Nacional de Estadística y Geografía (2017) Superficie agrícola sembrada, según cultivo y productos seleccionados. Instituto Nacional de Estadística y Geografía. Aguascalientes, México. https://www.inegi.org.mx/temas/agricultura/ (Marzo 2019).

Huerta-Espino J., R. P. Singh, S. A. Herrera-Foessel, J. B. Pérez-López and P. FigueroaLópez. (2009) First detection of virulence in Puccinia triticina to resistance genes $L r 27+L r 37$ present in durum wheat in Mexico. Plant Disease 93:110, https://doi.org/10.1094/PDIS-93-1-0110C

Pérez-López J. B., E. García-León, H. E. Villaseñor-Mir, R. P. Singh y K. Ammar (2017) Desarrollo de la epifitia de roya de la hoja causada por Puccinia triricina E. en la variedad de trigo cristalino Cirno C2008 durante el ciclo otoño-invierno 2016-2017 en el Bajo Río Mayo, Sonora. Revista Mexicana de Fitopatología 35(Supl. 2017):S39.

Cuadro 1. Rendimiento de grano $\left(\mathrm{t} \mathrm{ha}^{-1}\right)$ y reacción a royas de Conasist $\mathrm{C} 2015$ y variedades testigo (T); promedio de 98 ambientes en 10 entidades federativas de México en riego normal y limitado.

\begin{tabular}{lccccc}
\hline Variedad & RG & RRN & RRL & Rh & Ra \\
\hline Conasist C2015 & 5.1 & 5.8 & 4.4 & $0-10 R$ & $0-10 R$ \\
Anatoly C2011 (T) & 4.8 & 5.2 & 4.3 & 10MR-70S & $0-15 \mathrm{MR}$ \\
Cirno C2008 (T) & 4.6 & 5.2 & 4.0 & 10MR-30MS & 10MR-60MS \\
Gema C2004 (T) & 4.5 & 5.0 & 4.0 & 20MS-80S & $0-40 \mathrm{MS}$ \\
Rio Colorado C2000 (T) & 4.4 & 4.9 & 4.0 & 20MR-50MS & 20MR-70S \\
Movas C2011 (T) & 4.4 & 4.9 & 3.8 & $0-10 R$ & 20MR-80S \\
\hline
\end{tabular}

RG: rendimiento general de grano; RRN: rendimiento con riego normal, RRL: rendimiento con riego limitado, Rh: reacción a la roya de la hoja, Ra: reacción a la roya amarilla, R: resistente, MR: moderadamente resistente, MS: moderadamente susceptible, S: susceptible. 